\title{
A retrospective documentary review study of bacterial pathogen resistance to antimicrobials: a six months (July to December, 2016), at national health laboratory, Asmara, Eritrea
}

\begin{abstract}
Introduction: Antimicrobial resistance is defined as the resistance of an antimicrobial agent that is used to be effective against microbes but no longer works. Antimicrobial resistance is becoming a serious threat to global health as it is increasing worldwide requiring an integrated global action.
\end{abstract}

Objective: To assess the prevalence and identify antimicrobial resistance to commonly used drugs in relation to different types of samples from July to December, 2016 at National Health Laboratory, Asmara, Eritrea.

Methods: Retrospective documentary review study of laboratory results at National Health Laboratory, department of microbiology was applied to determine the pattern of antimicrobial resistance for bacterial isolates. All samples that brought to the microbiology laboratory were included in the study. Those samples that showed growth of isolates underwent for culture and sensitivity test against the commonly uses antimicrobial drugs. Statistical analysis of data was done using SPSS version 20.

Results: Out of 398 total samples, 149 samples had shown bacterial growth. Of these 149 samples: 48 were tested for E. Coli and found to be resistant to Ampicillin (42 (87.5\%)) and sensitive to chloramphenicol $(35(72.9 \%)) ; 20$ were tested for Klebsiella spp. found to be resistant to Ampicillin (15 (75\%)) and sensitive to Chloramphenicol in (16 (80\%)); 18 tested for Citrobacter spp. found to be resistant to Ampicillin in (18 (100\%)) and sensitive for Amikacin in (11 (61\%)); 11 tested for Pseudomonas spp. found to be resistant to majority of the drugs Ampicillin, Nalidixic acid. and Tetracycline, $(9(81.8 \%)$ each), Cephalexin and Cepfatizidime ( $8(72.7 \%)$ each), and Ceftriaxone $(7(63.6 \%))$; but they were found to be sensitive to Amikacin and Ciprofloxacin in $8(72.7 \%)$ and $7(63.6 \%)$, respectively. 8 of the samples tested for Proteus spp. were found to be resistant to Nitrofurantoin and Tetracycline ( $8(100 \%)$ each), where as they were observed to be sensitive to Amikacin $5(62.5 \%)$. 6 tested for Salmonella spp. and were also found to be sensitive to Amikacin and Ciprofloxacin in (4 (66.7\%), each), whereas resistant to Ampicillin $6(100 \%)$; Three samples were tested for Staphylococcus aureus and found to be resistant to most of the drugs; Nitrofurantoin $3(100 \%)$, and Gentamycin, Vancomycin, Oxacillin and Penicillin (2 (66.7\%) each); 35 samples were also tested for other isolates that include; Aeromonas Spp., Listeria Domsella, Candida Spp., Streptococcus Viridians, Kluvera Spp., Providencia Spp., Enterobacter Cloacae, Proteus Vulgaris, Chryseomonas Lutasa, Morganella Morganii, Neisseria Meningitidis, Acinetobacter Spp. and Pasteurella Maltocida; and showed a resistance to Cephalexin and Tetracycline (28 (80.1\%), each), Ampicillin 27 (77.1\%), Nalidixic Acid 26 (74.3\%) and Co- trimoxazole 21 (60\%).

Discussion and conclusion: The study results showed that most of the bacterial isolates were sensitive to Chloramphenicol Amikacin, Ciprofloxacin, Ceftriaxone and Gentamycin; whereas they were resistant to Cephalexin, Ampicillin, Ceftazidime, Nitrofurantoin, Cotrimoxazole, Nalidixic acid and Tetracycline. Therefore, the study recommends the practice of rational drug use to be permissible and continuous surveillance for antimicrobial drug sensitivity test to be done in order to assure appropriate drug administration for treating disease and reducing the emergence of new resistant strains of bacteria.

Keywords: culture, sensitivity, bacterial isolates, antimicrobial growth and resistance
Volume 5 Issue 3 - 2017

\author{
Yemane Seyoum,' Elias Teages Adgoy, ${ }^{2}$ \\ Kidane Siele, ${ }^{3}$ Mohammed Elfatih, ${ }^{4}$ Nesterab \\ Gebreleul $^{5}$ \\ 'Department of Internal Medicine, Orotta School of Medicine \\ and Dentistry, Asmara, Eritrea \\ ${ }^{2}$ Department of Community Medicine and Primary Health Care, \\ Orotta School of Medicine and Dentistry, Eritrea \\ ${ }^{3}$ Department of basic Sciences, Orotta School of Medicine and \\ Dentistry, Eritrea \\ ${ }^{4}$ Department of Microbiology, Orotta School of Medicine and \\ Dentistry, Eritrea \\ 5Department of Microbiology, National Health Laboratory, \\ Eritrea
}

Correspondence: Yemane Seyoum, Department of Interna Medicine, Orotta School of Medicine and Dentistry, Asmara, Eritrea,Email ssyemane@yahoo.com

Received: June 23, 2017 | Published: August II, 2017
Abbreviation: AMR, antimicrobial resistance; CLSI, clinical laboratory standards institute; CSF, cerebro-spinal fluid; ENF, eritrean national formulary; ENLM, eritrean national list of medicine; LMICs, low-income and middle-income countries; NHL, national health laboratory; SPSS, statistical package for social science

\section{Introduction}

Antimicrobials are natural, semi synthetic or synthetic substances that are capable of killing or inhibiting the growth of microbes. ${ }^{1}$ Antimicrobial resistance (AMR) simply means that the antimicrobial drugs that used to be effective against a particular microbe no longer 
work because of the change in the biological make-up of the microbe that makes it resistant to treatment. ${ }^{2,3}$ The problem occurs naturally, or when an infection is treated with an antimicrobial that kills only some of the microbes and making others resistant for the treatment by leaving to survive and multiply in the environment. ${ }^{2}$ In general there are many reasons for an increase of antimicrobial resistance that include: increasing consumption of antibiotics worldwide, as a result of treatment or diagnosis uncertainty physicians prescription of more antibiotics, significant access of antimicrobials agents in the use of as prophylaxis and self-administered treatment of infection, ${ }^{4,5}$ noncompliance with infection-control practice, increased duration of hospital stay, co-morbidities in hospitalized patients, frequent use of invasive devices and catheters, weak infection-control practices, transfer of colonized patients from hospital to hospital, grouping of patients in long-term-care facilities, use of antibiotic for animals and household tasks, and increasing national and international travel. ${ }^{6}$ The pattern of antimicrobial resistance differs from time to time, country to country and among hospitals within the same country. As a result the health care cost for patients with resistant infections is higher than non-resistant infections, due to longer duration of illness, additional tests and use of more expensive drugs. ${ }^{7}$ Over time, more and more of the resistant microbes remain in the environment, eventually leading to the emergence of new strains of disease-causing microbes that are partially or fully resistant to antimicrobial treatment. ${ }^{8,9}$ The level of antibiotic resistance depends on: the population of organisms that spontaneously acquire resistance to selective pressure from antibiotic use, rate of introduction of those resistant organisms into the health care settings, and the proportion of spread from person to person. ${ }^{10}$

Antimicrobial resistance is one of the serious problems due to new drug-resistant bacterial infections that are becoming more prevalent and becoming threat to the world public health care services and causing financial challenges ${ }^{11,12}$ (Coast, Smith \& Superbugs 1996; Smith 1999; Kohanski et al. 2010). Antimicrobial resistance is a global issue rather than a local or national. Data of regional and national antimicrobial resistance and trends of clinically important bacterial isolates are helpful for physicians in making decisions concerning the appropriate empirical treatment of various infections.

Therefore, this study was designed to explore the prevalence of antimicrobial resistance to the most common drugs that are used in the country for treating patients with different diagnosis of disease by doing culture and sensitivity test for different types of human samples that are collected at the National Health Laboratory (NHL) of Asmara, Eritrea. The study included the drugs that are listed in the Eritrean National Formulary ${ }^{13}$ and Eritrean National List of Medicine, ${ }^{14}$ that are available in the country as per to the government policy in matching to the Clinical Laboratory Standard Institute ${ }^{15}$ Guidelines; in order to provide a baseline information of sensitivity to these drugs. This study will have a great contribution for further research and will help also for health planners to design a strategy for effective and proper antimicrobial drug use.

\section{Objectives}

To assess and determine the prevalence of antimicrobial resistance to eighteen commonly used drugs in relation to different types of samples having various types of bacterial pathogen isolates from July $1^{\text {st }}$ to December $31^{\text {st }}, 2016$ at National Health Laboratory, Asmara, Eritrea.

\section{Methods and materials}

\section{Study setting and design}

A retrospective cross-sectional study was conducted at National Health Laboratory (NHL), Asmara, Eritrea. The study was done based on retrospective record review of patients from July $1^{\text {st }}$ to December $31^{\text {st }}, 2016$; on antimicrobial susceptibility tests performed on bacterial isolates in the National Health Laboratory Department of Microbiology Asmara, Eritrea.

\section{Study participants and study period}

All samples that were brought to the study area were included. Those samples that showed growth of bacterial isolates that underwent sensitivity test against the commonly used antimicrobial drugs based on the ENF and ENLM that correspond with the CLSI as well.

\section{Data collection}

Data related to the variables of interest were collected, based on the common tests that were performed in the Microbiology Laboratory. Primarily qualitative data reflecting socio-demographic and resistance level of microbes (resistant, intermediate, or susceptible) was collected. The collected data was entered into an Excel. Variables of interest included the first isolate from each specimen of patient such as ascitic fluid, pleural fluid, blood, Cerebro - spinal fluid (CSF), synovial fluid, nasal swab, sputum, seminal fluid, urine, urethral discharge, and vaginal discharge, stool, and pus. The specimens were analyzed based on the isolate and with different antibiotics susceptibility profiles.

\section{Data processing and analysis}

The study used SPSS version 20 for statistical analysis of the data collected.

\section{Ethical consideration}

The study obtained permission from Ministry of Health ethical and research committee, and National Health Laboratory and all records with in the study period were checked. The procedure of the study was very confidential in that the records were checked by the researchers only.

\section{Results}

The number of specimens that were collected at National Health Laboratory, Asmara, Eritrea, from July $1^{\text {st }}$ to December $31^{\text {st }}$, 2016 included a total of four hundreds of different types. Those samples that were included in the review were tested by using Mueller Hintone Agar for culture and sensitivity test of the antimicrobials. Therefore, out of these four hundred specimens that under went for culture and sensitivity test the number of adults was higher 67.75 percent $(n=271)$ as compared to the number of children32.25 percent $(n=129)$. The number of females $(n=210)$ was also higher than that of males $(\mathrm{n}=190)$ (Table 1). The study population had a mean age of 4.43 and 42.35years for children and adults, respectively (Table 1).

Table I Demographic characteristics of patients for culture and sensitivity test

\begin{tabular}{|c|c|c|c|c|}
\hline & & Children (<18Years) & Adults (> I 8Years) & Total \\
\hline \multirow{2}{*}{ Gender } & Male & $50(26.3 \%)$ & 140 (73.7\%) & 190 \\
\hline & Female & $79(37.6 \%)$ & 131 (62.4\%) & 210 \\
\hline
\end{tabular}


Table Continued...

\begin{tabular}{|c|c|c|c|c|}
\hline & & Children (<18Years) & Adults (> I 8Years) & Total \\
\hline & Total Patients Number & $129(32.2 \%)$ & $27 \mathrm{I}(67.75 \%)$ & 400 \\
\hline Mean age in years (standard deviation) & & $4.43(4.8)$ & $42.35(16.2)$ & $30.12(22.4)$ \\
\hline
\end{tabular}

\section{Sample types with presence or absence of growth}

The specimen were categorized based on their type as ascitic fluid, pleural fluid, blood, CSF, synovial fluid, nasal swab, sputum, seminal fluid, urine, urethral discharge, vaginal discharge, stool, and pus.

The most frequent specimen with 60.05 Percent $(n=239)$ was urine and the least were synovial fluid and sputum with 0.25 percent $(n=1)$ each. In majority of the samples growth of bacteria was not observed having general result of non - growth 62.6percent $(n=249)$ as compared to 37.4 percent $(\mathrm{n}=149)$ growth (Table 2$)$

Table 2 Frequency of samples and distribution of presence or absence of growth of various isolates

\begin{tabular}{llll}
\hline \multirow{2}{*}{ Sample type } & \multicolumn{3}{l}{ Frequency of presence or absence of growth } \\
\cline { 2 - 4 } & No $(\%)$ & Yes (\%) & Total Samples \\
\hline Ascitic Fluid & $3(75 \%)$ & $\mathrm{I}(25 \%)$ & $4(\%)$ \\
Pleural Fluid & $4(100 \%)$ & $0(0 \%)$ & $4(\%)$ \\
Blood & $54(96.4 \%)$ & $2(3.6 \%)$ & $56(14.07 \%)$ \\
CSF & $16(88.9 \%)$ & $2(11.1 \%)$ & $18(4.52 \%)$ \\
Synovial Fluid & $\mathrm{I}(100 \%)$ & $0(0 \%)$ & $\mathrm{I}(0.25 \%)$ \\
Nasal Swab & $0(0 \%)$ & $2(100 \%)$ & $2(0.5 \%)$ \\
Sputum & $0(0 \%)$ & $\mathrm{I}(100 \%)$ & $\mathrm{I}(0.25 \%)$ \\
Seminal Fluid & $\mathrm{I}(50 \%)$ & $\mathrm{I}(50 \%)$ & $2(0.5 \%)$ \\
Urine & $130(54.4 \%)$ & $109(45.6 \%)$ & $239(60.05 \%)$ \\
Urethral Discharge & $3(75 \%)$ & $\mathrm{I}(25 \%)$ & $4(\%)$ \\
Vaginal Discharge & $22(46.8 \%)$ & $25(53.2 \%)$ & $47(\mathrm{II} .8 \%)$ \\
Stool & $9(90 \%)$ & $\mathrm{I}(10 \%)$ & $10(2.5 \%)$ \\
Pus & $6(60 \%)$ & $4(40 \%)$ & $10(2.5 \%)$ \\
Total & $249(62.6 \%)$ & $149(37.4 \%)$ & 398
\end{tabular}

\section{Antimicrobials susceptibility pattern of isolates}

The total samples $(n=149)$ that showed growth were tested for sensitivity against different drugs. All (eighteen) the commonly used antibiotics that went through culture and sensitivity test of different samples for various pathogens in the study period are abbreviated as per to the Clinical laboratory Standards Institute (CLSI), (Table 3) (Table 4). According to the results out of forty eight samples that were tested for E. Coli, it was found to be resistant to Ampicillin (42 $(87.5 \%))$ and sensitive to chloramphenicol $(35(72.9 \%))$.

Twenty samples showing growth of Klebsiella spp. were also tested and it was found to be resistant to Ampicillin (15(75\%)) and sensitive to Chloramphenicol in $(16(80 \%))$. Out of eighteen samples of Citrobacter spp. a resistance to Ampicillin in (18(100\%)) and sensitivity to Amikacin in (11(61\%)) (Table 3). Out of eleven samples, Pseudomonas spp. were also found to be resistant to the majority of the drugs; Ampicillin, Nalidixic acid and Tetracycline, (9(81.8\%) each), Cephalexin and Ceftazidime (8 (72.7\%) each), and Ceftriaxone (7(63.6\%)); but they were found to be sensitive to Amikacin and Ciprofloxacinfor 8 (72.7\%) and 7(63.6\%), respectively (Table 3) (Table 4). Eight samples had culture and sensitivity test for Proteus spp. in which Nitrofurantoin and Tetracycline were found to be resistant (8(100\%) each), whereas sensitive to Amikacin 5(62.5\%) (Table 3). Out of six samples that showed growth for Salmonella spp. sensitivity was observed to Amikacin and Ciprofloxacin in (4(66.7\%) each), whereas resistant to Ampicillin 6(100\%). Out of three samples Staphylococcus aureus was observed to be resistant to most of the drugs; Nitrofurantoin $3(100 \%)$, and Gentamycin, Vancomycin, Oxacillin and Penicillin in (2(66.7\%), each) (Table 3) (Table 4).There were also other isolates that include: Aeromonas Spp, Listeria Domsella, Candida Spp., Streptococcus Viridians, Kluvera Spp., Providencia Spp., Enterobacter Cloacae, Proteus Vulgaris, Chryseomonas Lutasa, Morganella Morganii, Neisseria Meningitidis, Acinetobacter Spp. and Pasteurella Maltocida from the samples that were tested for the antimicrobial susceptibility and they were observed to be resistant to Cephalexin and Tetracycline (28(80.1\%), each). Ampicillin 27(77.1\%) Nalidixic acid 26(74.3\%) and Co - trimoxazole 21(60\%) (Table 3) (Table 4).

Table 3 Antibiotic susceptibility pattern of twelve most frequent bacterial isolates

\begin{tabular}{|c|c|c|c|c|c|c|c|c|c|c|c|c|c|c|}
\hline \multirow{2}{*}{$\begin{array}{c}\text { Bacterial } \\
\text { Isolate }\end{array}$} & \multirow{2}{*}{ TS } & \multirow{2}{*}{ Pat } & \multicolumn{12}{|c|}{ Antimicrobial Agents, n (\%) } \\
\hline & & & AK & AMX & $\mathrm{CN}$ & CAZ & CRO & CIP & COT & GM & NIT & NA & TE & c \\
\hline \multirow{3}{*}{ E. Coli } & \multirow{3}{*}{48} & 1 & $\begin{array}{c}2 \\
(4.2 \%)\end{array}$ & $\begin{array}{c}3 \\
(6.2 \%)\end{array}$ & $\begin{array}{c}0 \\
(0 \%)\end{array}$ & $\begin{array}{c}4 \\
(8.3 \%)\end{array}$ & $\begin{array}{c}0 \\
(0 \%)\end{array}$ & $\begin{array}{c}0 \\
(0 \%)\end{array}$ & $\begin{array}{c}0 \\
(0 \%)\end{array}$ & $\begin{array}{c}0 \\
(0 \%)\end{array}$ & $\begin{array}{c}2 \\
(4.2 \%)\end{array}$ & $\begin{array}{c}1 \\
(2.1 \%)\end{array}$ & $\begin{array}{c}6 \\
(12.5 \%)\end{array}$ & $\begin{array}{c}1 \\
(2.1 \%)\end{array}$ \\
\hline & & R & $\begin{array}{c}5 \\
(10.4 \%)\end{array}$ & $\begin{array}{c}42 \\
(87.5 \%)\end{array}$ & $\begin{array}{c}35 \\
(72.9 \%)\end{array}$ & $\begin{array}{c}26 \\
(54.2 \%)\end{array}$ & $\begin{array}{c}20 \\
(41.7 \%)\end{array}$ & $\begin{array}{c}19 \\
(39.6 \%)\end{array}$ & $\begin{array}{c}23 \\
(47.9 \%)\end{array}$ & $\begin{array}{c}12 \\
(25 \%)\end{array}$ & $\begin{array}{c}16 \\
(33.3 \%)\end{array}$ & $\begin{array}{c}28 \\
(58.3 \%)\end{array}$ & $\begin{array}{c}27 \\
(56.3 \%)\end{array}$ & $\begin{array}{c}9 \\
(18.6 \%)\end{array}$ \\
\hline & & s & $\begin{array}{c}24 \\
(50 \%)\end{array}$ & $\begin{array}{c}3 \\
(6.5 \%)\end{array}$ & $\begin{array}{c}0 \\
(0 \%)\end{array}$ & $\begin{array}{c}13 \\
(27.1 \%)\end{array}$ & $\begin{array}{c}27 \\
(56.3 \%)\end{array}$ & $\begin{array}{c}24 \\
(50 \%)\end{array}$ & $\begin{array}{c}15 \\
(31.3 \%)\end{array}$ & $\begin{array}{c}34 \\
(70 \%)\end{array}$ & $\begin{array}{c}14 \\
(29.2 \%)\end{array}$ & $\begin{array}{c}14 \\
(29.2 \%)\end{array}$ & $\begin{array}{c}13 \\
(27.1 \%)\end{array}$ & $\begin{array}{c}35 \\
(72.9 \%)\end{array}$ \\
\hline \multirow{3}{*}{ Klebsiella spp. } & \multirow{3}{*}{20} & 1 & $\begin{array}{c}0 \\
(0 \%)\end{array}$ & $\begin{array}{c}1 \\
(5 \%)\end{array}$ & $\begin{array}{c}1 \\
(5 \%)\end{array}$ & $\begin{array}{c}3 \\
(15 \%)\end{array}$ & $\begin{array}{c}2 \\
(10 \%)\end{array}$ & $\begin{array}{c}0 \\
(0 \%)\end{array}$ & $\begin{array}{c}2 \\
(10 \%)\end{array}$ & $\begin{array}{c}0 \\
(0 \%)\end{array}$ & $\begin{array}{c}0 \\
(0 \%)\end{array}$ & $\begin{array}{c}1 \\
(10 \%)\end{array}$ & $\begin{array}{c}3 \\
(15 \%)\end{array}$ & $\begin{array}{c}0 \\
(0 \%)\end{array}$ \\
\hline & & R & $\begin{array}{c}2 \\
(10 \%)\end{array}$ & $\begin{array}{c}15 \\
(75 \%)\end{array}$ & $\begin{array}{c}13 \\
(65 \%)\end{array}$ & $\begin{array}{c}14 \\
(70 \%)\end{array}$ & $\begin{array}{c}11 \\
(55 \%)\end{array}$ & $\begin{array}{c}8 \\
(40 \%)\end{array}$ & $\begin{array}{c}9 \\
(45 \%)\end{array}$ & $\begin{array}{c}10 \\
(50 \%)\end{array}$ & $\begin{array}{c}11 \\
(55 \%)\end{array}$ & $\begin{array}{c}13 \\
(65 \%)\end{array}$ & $\begin{array}{c}13 \\
(65 \%)\end{array}$ & $\begin{array}{c}3 \\
(15 \%)\end{array}$ \\
\hline & & s & $\begin{array}{c}12 \\
(60 \%)\end{array}$ & $\begin{array}{c}3 \\
(15 \%)\end{array}$ & $\begin{array}{c}0 \\
(0 \%)\end{array}$ & $\begin{array}{c}2 \\
(10 \%)\end{array}$ & $\begin{array}{c}7 \\
(35 \%)\end{array}$ & $\begin{array}{c}8 \\
(40 \%)\end{array}$ & $\begin{array}{c}8 \\
(40 \%)\end{array}$ & $\begin{array}{c}9 \\
(45 \%)\end{array}$ & $\begin{array}{c}3 \\
(15 \%)\end{array}$ & $\begin{array}{c}6 \\
(30 \%)\end{array}$ & $\begin{array}{c}4 \\
(20 \%)\end{array}$ & $\begin{array}{c}16 \\
(80 \%)\end{array}$ \\
\hline \multirow{3}{*}{ Citrobacter spp. } & \multirow{3}{*}{18} & 1 & $\begin{array}{c}2 \\
(11.1 \%)\end{array}$ & $\begin{array}{c}0 \\
(0 \%)\end{array}$ & $\begin{array}{c}0 \\
(0 \%)\end{array}$ & $\begin{array}{c}3 \\
(16.7 \%)\end{array}$ & $\begin{array}{c}1 \\
(5.6 \%)\end{array}$ & $\begin{array}{c}1 \\
(5.6 \%)\end{array}$ & $\begin{array}{c}0 \\
(0 \%)\end{array}$ & $\begin{array}{c}0 \\
(0 \%)\end{array}$ & $\begin{array}{c}1 \\
(5.6 \%)\end{array}$ & $\begin{array}{c}\text { I } \\
(5.6 \%)\end{array}$ & $\begin{array}{c}1 \\
(5.6 \%)\end{array}$ & $\begin{array}{c}1 \\
(5.6 \%)\end{array}$ \\
\hline & & $\mathrm{R}$ & $\begin{array}{c}2 \\
(11.1 \%)\end{array}$ & $\begin{array}{c}18 \\
(100 \%)\end{array}$ & $\begin{array}{c}16 \\
(88.9 \%)\end{array}$ & $\begin{array}{c}9 \\
(50 \%)\end{array}$ & $\begin{array}{c}6 \\
(33.3 \%)\end{array}$ & $\begin{array}{c}6 \\
(33.3 \%)\end{array}$ & $\begin{array}{c}8 \\
(44.4 \%)\end{array}$ & $\begin{array}{c}6 \\
(33.3 \%)\end{array}$ & $\begin{array}{c}6 \\
(33.3 \%)\end{array}$ & $\begin{array}{c}11 \\
(61.1 \%)\end{array}$ & $\begin{array}{c}9 \\
(50 \%)\end{array}$ & $\begin{array}{c}5 \\
(27.8 \%)\end{array}$ \\
\hline & & s & $\begin{array}{c}11 \\
(61.1 \%)\end{array}$ & $\begin{array}{c}0 \\
(0 \%)\end{array}$ & $\begin{array}{c}0 \\
(0 \%)\end{array}$ & $\begin{array}{c}4 \\
(22.2 \%)\end{array}$ & $\begin{array}{c}11 \\
(61.1 \%)\end{array}$ & $\begin{array}{c}10 \\
(55.6 \%)\end{array}$ & $\begin{array}{c}7 \\
(38.9 \%)\end{array}$ & $\begin{array}{c}10 \\
(55.6 \%)\end{array}$ & $\begin{array}{c}4 \\
(22.2 \%)\end{array}$ & $\begin{array}{c}6 \\
(33.3 \%)\end{array}$ & $\begin{array}{c}7 \\
(38.9 \%)\end{array}$ & $\begin{array}{c}10 \\
(55.6 \%)\end{array}$ \\
\hline
\end{tabular}

Citation: Seyoum Y,Adgoy ET, Siele K, et al.A retrospective documentary review study of bacterial pathogen resistance to antimicrobials: a six months (July to December, 20 I6), at national health laboratory, Asmara, Eritrea.J Bacteriol Mycol Open Access. 20 I7;5(3):287-292. DOI: I0.15406/jbmoa.20I7.05.00I 33 
Table Continued....

\begin{tabular}{|c|c|c|c|c|c|c|c|c|c|c|c|c|c|c|}
\hline \multirow{2}{*}{$\begin{array}{l}\text { Bacterial } \\
\text { Isolate }\end{array}$} & \multirow{2}{*}{ TS } & \multirow{2}{*}{ Pat } & \multicolumn{12}{|c|}{ Antimicrobial Agents, $n$ (\%) } \\
\hline & & & AK & $A M X$ & CN & CAZ & CRO & CIP & СОт & GM & NIT & NA & TE & C \\
\hline \multirow{3}{*}{$\begin{array}{c}\text { Pseudomonas } \\
\text { spp. }\end{array}$} & \multirow{3}{*}{11} & 1 & $\begin{array}{c}0 \\
(0 \%)\end{array}$ & $\begin{array}{c}0 \\
(0 \%)\end{array}$ & $\begin{array}{c}0 \\
(0 \%)\end{array}$ & $\begin{array}{c}0 \\
0 \%)\end{array}$ & $\begin{array}{c}1 \\
(9.1 \%)\end{array}$ & $\begin{array}{c}0 \\
(0 \%)\end{array}$ & $\begin{array}{c}1 \\
(9.1 \%)\end{array}$ & $\begin{array}{c}1 \\
(9.1 \%)\end{array}$ & $\begin{array}{c}0 \\
(0 \%)\end{array}$ & $\begin{array}{c}1 \\
(9.1 \%)\end{array}$ & $\begin{array}{c}0 \\
(0 \%)\end{array}$ & $\begin{array}{c}3 \\
(27.3 \%)\end{array}$ \\
\hline & & $\mathrm{R}$ & $\begin{array}{c}1 \\
(9.1 \%)\end{array}$ & $\begin{array}{c}9 \\
(81.8 \%)\end{array}$ & $\begin{array}{c}8 \\
(73 \%)\end{array}$ & $\begin{array}{c}8 \\
(73 \%)\end{array}$ & $\begin{array}{c}7 \\
(63.6 \%)\end{array}$ & $\begin{array}{c}4 \\
(36.4 \%)\end{array}$ & $\begin{array}{c}7 \\
(63.6 \%)\end{array}$ & $\begin{array}{c}5 \\
(45.5 \%)\end{array}$ & $\begin{array}{c}8 \\
(73 \%)\end{array}$ & $\begin{array}{c}9 \\
(81.8 \%)\end{array}$ & $\begin{array}{c}9 \\
(81.8 \%)\end{array}$ & $\begin{array}{c}5 \\
(45.5 \%)\end{array}$ \\
\hline & & s & $\begin{array}{c}8 \\
(73 \%)\end{array}$ & $\begin{array}{c}1 \\
(9.1 \%)\end{array}$ & $\begin{array}{c}1 \\
(9.1 \%)\end{array}$ & $\begin{array}{c}2 \\
(18.2 \%)\end{array}$ & $\begin{array}{c}3 \\
(27.3 \%)\end{array}$ & $\begin{array}{c}7 \\
(63.6 \%)\end{array}$ & $\begin{array}{c}2 \\
(18.2 \%)\end{array}$ & $\begin{array}{c}4 \\
(36.4 \%)\end{array}$ & $\begin{array}{c}1 \\
(9.1 \%)\end{array}$ & $\begin{array}{c}1 \\
(9.1 \%)\end{array}$ & $\begin{array}{c}2 \\
(18.2 \%)\end{array}$ & $\begin{array}{c}0 \\
(0 \%)\end{array}$ \\
\hline \multirow{3}{*}{ Proteus spp. } & \multirow{3}{*}{8} & 1 & $\begin{array}{c}2 \\
(25 \%)\end{array}$ & $\begin{array}{c}0 \\
(0 \%)\end{array}$ & $\begin{array}{c}0 \\
(0 \%)\end{array}$ & $\begin{array}{c}0 \\
(0 \%)\end{array}$ & $\begin{array}{c}0 \\
(0 \%)\end{array}$ & $\begin{array}{c}1 \\
(12.5 \%)\end{array}$ & $\begin{array}{c}0 \\
(0 \%)\end{array}$ & $\begin{array}{c}0 \\
(0 \%)\end{array}$ & $\begin{array}{c}0 \\
(0 \%)\end{array}$ & $\begin{array}{c}0 \\
(0 \%)\end{array}$ & $\begin{array}{c}0 \\
(0 \%)\end{array}$ & $\begin{array}{c}0 \\
(0 \%)\end{array}$ \\
\hline & & $\mathrm{R}$ & $\begin{array}{c}1 \\
(12.5 \%)\end{array}$ & $\begin{array}{c}6 \\
(75 \%)\end{array}$ & $\begin{array}{c}6 \\
(75 \%)\end{array}$ & $\begin{array}{c}6 \\
(75 \%)\end{array}$ & $\begin{array}{c}6 \\
(75 \%)\end{array}$ & $\begin{array}{c}6 \\
(75 \%)\end{array}$ & $\begin{array}{c}7 \\
(87.5 \%)\end{array}$ & $\begin{array}{c}6 \\
(75 \%)\end{array}$ & $\begin{array}{c}8 \\
(100 \%)\end{array}$ & $\begin{array}{c}6 \\
(75 \%)\end{array}$ & $\begin{array}{c}8 \\
(100 \%)\end{array}$ & $\begin{array}{c}6 \\
(75 \%)\end{array}$ \\
\hline & & s & $\begin{array}{c}5 \\
(62.5 \%)\end{array}$ & $\begin{array}{c}0 \\
(0 \%)\end{array}$ & $\begin{array}{c}0 \\
(0 \%)\end{array}$ & $\begin{array}{c}1 \\
(12.5 \%)\end{array}$ & $\begin{array}{c}2 \\
(25 \%)\end{array}$ & $\begin{array}{c}1 \\
(12.5 \%)\end{array}$ & $\begin{array}{c}1 \\
(12.5 \%)\end{array}$ & $\begin{array}{c}2 \\
(25 \%)\end{array}$ & $\begin{array}{c}0 \\
(0 \%)\end{array}$ & $\begin{array}{c}1 \\
(12.5 \%)\end{array}$ & $\begin{array}{c}0 \\
(0 \%)\end{array}$ & $\begin{array}{c}1 \\
(12.5 \%)\end{array}$ \\
\hline \multirow{3}{*}{ Salmonella spp. } & \multirow{3}{*}{6} & 1 & $\begin{array}{c}0 \\
(0 \%)\end{array}$ & $\begin{array}{c}0 \\
(0 \%)\end{array}$ & $\begin{array}{c}0 \\
(0 \%)\end{array}$ & $\begin{array}{c}0 \\
(0 \%)\end{array}$ & $\begin{array}{c}1 \\
(16.7 \%)\end{array}$ & $\begin{array}{c}0 \\
(0 \%)\end{array}$ & $\begin{array}{c}0 \\
(0 \%)\end{array}$ & $\begin{array}{c}0 \\
(0 \%)\end{array}$ & $\begin{array}{c}1 \\
(16.7 \%)\end{array}$ & $\begin{array}{c}0 \\
(0 \%)\end{array}$ & $\begin{array}{c}1 \\
(16.7 \%)\end{array}$ & $\begin{array}{c}0 \\
(0 \%)\end{array}$ \\
\hline & & $\mathrm{R}$ & $\begin{array}{c}1 \\
(16.7 \%)\end{array}$ & $\begin{array}{c}6 \\
(100 \%)\end{array}$ & $\begin{array}{c}3 \\
(50 \%)\end{array}$ & $\begin{array}{c}4 \\
(66.7 \%)\end{array}$ & $\begin{array}{c}3 \\
(50 \%)\end{array}$ & $\begin{array}{c}1 \\
(16.7 \%)\end{array}$ & $\begin{array}{c}3 \\
(50 \%)\end{array}$ & $\begin{array}{c}1 \\
(16.7 \%)\end{array}$ & $\begin{array}{c}2 \\
(33.3 \%)\end{array}$ & $\begin{array}{c}3 \\
(50 \%)\end{array}$ & $\begin{array}{c}3 \\
(50 \%)\end{array}$ & $\begin{array}{c}3 \\
(50 \%)\end{array}$ \\
\hline & & s & $\begin{array}{c}4 \\
(66.7 \%)\end{array}$ & $\begin{array}{c}0 \\
(0 \%)\end{array}$ & $\begin{array}{c}1 \\
(16.7 \%)\end{array}$ & $\begin{array}{c}0 \\
(0 \%)\end{array}$ & $\begin{array}{c}1 \\
(16.7 \%)\end{array}$ & $\begin{array}{c}4 \\
(66.7 \%)\end{array}$ & $\begin{array}{c}1 \\
(16.7 \%)\end{array}$ & $\begin{array}{c}2 \\
(33.3 \%)\end{array}$ & $\begin{array}{c}1 \\
(16.7 \%)\end{array}$ & $\begin{array}{c}2 \\
(33.3 \%)\end{array}$ & $\begin{array}{c}2 \\
(33.3 \%)\end{array}$ & $\begin{array}{c}3 \\
(50 \%)\end{array}$ \\
\hline \multirow{3}{*}{ S. aureus } & \multirow{3}{*}{3} & 1 & $\begin{array}{c}0 \\
(0 \%)\end{array}$ & $\begin{array}{c}0 \\
(0 \%)\end{array}$ & $\begin{array}{c}0 \\
(0 \%)\end{array}$ & $\begin{array}{c}0 \\
(0 \%)\end{array}$ & $\begin{array}{c}0 \\
(0 \%)\end{array}$ & $\begin{array}{c}2 \\
(66.7 \%)\end{array}$ & $\begin{array}{c}0 \\
(0 \%)\end{array}$ & $\begin{array}{c}0 \\
(0 \%)\end{array}$ & $\begin{array}{c}0 \\
(0 \%)\end{array}$ & $\begin{array}{c}0 \\
(0 \%)\end{array}$ & $\begin{array}{c}\text { I } \\
(33.3 \%)\end{array}$ & $\begin{array}{c}0 \\
(0 \%)\end{array}$ \\
\hline & & $\mathrm{R}$ & $\begin{array}{c}0 \\
(0 \%)\end{array}$ & $\begin{array}{c}0 \\
(0 \%)\end{array}$ & $\begin{array}{c}0 \\
(0 \%)\end{array}$ & $\begin{array}{c}0 \\
(0 \%)\end{array}$ & $\begin{array}{c}0 \\
(0 \%)\end{array}$ & $\begin{array}{c}0 \\
(0 \%)\end{array}$ & $\begin{array}{c}1 \\
(33.3 \%)\end{array}$ & $\begin{array}{c}2 \\
(66.7 \%)\end{array}$ & $\begin{array}{c}3 \\
(100 \%)\end{array}$ & $\begin{array}{c}0 \\
(0 \%)\end{array}$ & $\begin{array}{c}\text { I } \\
(33.3 \%)\end{array}$ & $\begin{array}{c}1 \\
(33.3 \%)\end{array}$ \\
\hline & & s & $\begin{array}{c}0 \\
(0 \%)\end{array}$ & $\begin{array}{c}0 \\
(0 \%)\end{array}$ & $\begin{array}{c}0 \\
(0 \%)\end{array}$ & $\begin{array}{c}0 \\
(0 \%)\end{array}$ & $\begin{array}{c}0 \\
(0 \%)\end{array}$ & $\begin{array}{c}1 \\
(33.3 \%)\end{array}$ & $\begin{array}{c}2 \\
(66.7 \%)\end{array}$ & $\begin{array}{c}1 \\
(33.3 \%)\end{array}$ & $\begin{array}{c}0 \\
(0 \%)\end{array}$ & $\begin{array}{c}0 \\
(0 \%)\end{array}$ & $\begin{array}{c}0 \\
(0 \%)\end{array}$ & $2(66.7 \%)$ \\
\hline \multirow{3}{*}{ Other isolates* } & \multirow{3}{*}{35} & 1 & $\begin{array}{c}2 \\
(5.7 \%)\end{array}$ & $\begin{array}{c}2 \\
(5.7 \%)\end{array}$ & $\begin{array}{c}0 \\
(0 \%)\end{array}$ & $\begin{array}{c}3 \\
(8.6 \%)\end{array}$ & $\begin{array}{c}2 \\
(5.7 \%)\end{array}$ & $\begin{array}{c}0 \\
(0 \%)\end{array}$ & $\begin{array}{c}0 \\
(0 \%)\end{array}$ & $\begin{array}{c}0 \\
(0 \%)\end{array}$ & $\begin{array}{c}2 \\
(5.7 \%)\end{array}$ & $\begin{array}{c}1 \\
(2.9 \%)\end{array}$ & $\begin{array}{c}0 \\
(0 \%)\end{array}$ & $\mathrm{I}(2.9 \%)$ \\
\hline & & $\mathrm{R}$ & $\begin{array}{c}1 \\
(2.9 \%)\end{array}$ & $\begin{array}{c}27 \\
(77.1 \%)\end{array}$ & $\begin{array}{c}28 \\
(80 \%)\end{array}$ & $\begin{array}{c}21 \\
(60 \%)\end{array}$ & $\begin{array}{c}12 \\
(34.3 \%)\end{array}$ & $\begin{array}{c}19 \\
(54.3 \%)\end{array}$ & $\begin{array}{c}21 \\
(60 \%)\end{array}$ & $\begin{array}{c}12 \\
(34.3 \%)\end{array}$ & $\begin{array}{c}19 \\
(54.3 \%)\end{array}$ & $\begin{array}{c}26 \\
(74.3 \%)\end{array}$ & $\begin{array}{c}28 \\
(80 \%)\end{array}$ & $15(42.9 \%)$ \\
\hline & & s & 24 & $\begin{array}{c}5 \\
(14.3 \%)\end{array}$ & $\begin{array}{c}2 \\
(5.7 \%)\end{array}$ & $\begin{array}{c}5 \\
(14.3 \%)\end{array}$ & $\begin{array}{c}16 \\
(45.7 \%)\end{array}$ & $\begin{array}{c}16 \\
(45.7 \%)\end{array}$ & $\begin{array}{c}12 \\
(34.3 \%)\end{array}$ & $\begin{array}{c}19 \\
(54.3 \%)\end{array}$ & $\begin{array}{c}7 \\
(20 \%)\end{array}$ & $\begin{array}{c}6 \\
(17.1 \%)\end{array}$ & $\begin{array}{c}9 \\
(25.7 \%)\end{array}$ & $17(48.6 \%)$ \\
\hline
\end{tabular}

TS, total sample; Pat, pattern; I, intermediate; R, resistant; S, sensitive; AK, amikacin; AMX, ampicillin; CN, cephalexin; CAZ, ceftazidime; CRO, ceftriaxone; CIP, ciprofloxacin; COT, co -trimoxazole; GM, gentamycin; NIT, nitofurantoin; NA, nalidixic Acid; TE, tetracycline; C, chloramphenicol

Table 4 Antibiotic susceptibility pattern of the six most frequent bacterial isolates

\begin{tabular}{lllllllll}
\hline \multirow{2}{*}{ Bacterial isolate } & \multirow{2}{*}{ TS } & Pat & \multicolumn{2}{l}{ Antimicrobial agents, n (\%) } \\
\cline { 4 - 9 } & & & E & RA & VA & CC & OX & P \\
\hline \multirow{2}{*}{ S. aureus } & & $\mathrm{I}$ & $\mathrm{I}(33.3 \%)$ & $0(0 \%)$ & $0(0 \%)$ & $0(0 \%)$ & $00(0 \%)$ & $0(0 \%)$ \\
& 3 & $\mathrm{R}$ & $0(0 \%)$ & $0(0 \%)$ & $2(66.7 \%)$ & $0(0 \%)$ & $2(66.7 \%)$ & $2(66.7 \%)$ \\
& & $\mathrm{S}$ & $2(66.7 \%)$ & $3(100 \%)$ & $\mathrm{I}(33.3 \%)$ & $0(0 \%)$ & $\mathrm{I}(33.3 \%)$ & $0(0 \%)$ \\
Others* & & $\mathrm{I}$ & $0(0 \%)$ & $0(0 \%)$ & $0(0 \%)$ & $0(0 \%)$ & $0(0 \%)$ & $0(0 \%)$ \\
& 35 & $\mathrm{R}$ & $\mathrm{I}(2.9 \%)$ & $2(5.7 \%)$ & $3(8.6 \%)$ & $0(0 \%)$ & $0(0 \%)$ & $\mathrm{I}(2.9 \%)$ \\
& & $\mathrm{S}$ & $0(0 \%)$ & $\mathrm{I}(2.9 \%)$ & $0(0 \%)$ & $0(0 \%)$ & $0(0 \%)$ & $0(0 \%)$
\end{tabular}

TS, total sample; Pat, pattern; I, intermediate; R, resistant; S, sensitive; E, erythromycin; RA, rifampicin; VA, vancomycin; CC, clindamycin; OX, oxacillin; P, penicillin

Others* This are bacterial isolates that has been detected in very low number and include: Aeromonas Spp., Listeria Domsella, Candida Spp., Streptococcus Viridians, Kluvera Spp., Providencia Spp., Enterobacter Cloacae, Proteus Vulgaris, Chryseomonas Lutasa, Morganella Morganii, Neisseria Meningitidis, Acinetobacter Spp. and Pasteurella Maltocida.

\section{Discussion}

The study results showed a lower rate of an overall growth of bacteria which is 37.4 percent ( $\mathrm{n}=149)$. Similar cross-sectional study that was done in Iran, from July 2006 to June 2007, also documented a growth for Klebsiella spp. (22.4\%), Staphylococcus aureus (12\%), Citrobacter spp. (8.8\%), and Escherichia coli (8.2\%), being the most frequent microorganisms. ${ }^{16}$ The similarity and differences of the growth rate can be due to difference in the use of equipment, material and setting services related to culture and sensitivity test preparations and. supply management. Like many other studies this study also documented E.coli isolates as the most prevalent 32.2 percent $(\mathrm{n}=48)$ and it was observed to be highly sensitive to chloramphenicol, Gentamycin and Ceftriaxone with a sensitivity rate of 72.9 percent, 70 percent and 56.3 percent, respectively; but resistant to Ampicillin and Cephalexin in 87.5 percent and 72.9 percent. This resistance specifically to Ampicillin, tetracycline, Co - trimoxazole and amoxicillin can be mainly related to the self-administration of common antibiotic drugs because of easy access as routing drugs for therapeutic interventions and as commonly used without prescription even for animals. Antibiotic use continued to be higher in high income countries the agricultural activities in the community have contributed 
to selection pressure that has sustained resistant strains, forcing to too costly and broad-spectrum antibiotics use.

In developing low-income and middle-income countries (LMICs) antibiotic use is increasing with rising incomes, high rates of hospitalization, and high prevalence of hospital infections. ${ }^{17}$ A Retrospective study in Lebanese hospitals also documented a sensitivity of 97.2 percent and 71.7 percent, for Amikacin and Gentamycin, respectively. ${ }^{18}$ A study that was conducted in Ethiopia by Dessie, ${ }^{19}$ for susceptibility test of isolates from surgical site infection documented a similar sensitivity test of 75 percent for Chloramphenicol and resistance for Ampicillin, and Tetracycline in 95.8 and 83.3 percent, respectively. ${ }^{19}$ A similar study in Kenya documented a resistance to Ampicillin and Co-trimoxazole of 60 percent, each. ${ }^{20}$ Another study in Ethiopia also documented a resistance to Ampicillin $(80 \%)$ and sensitivity to Gentamycin (66\%), Ceftriaxone (53.1\%), and Chloramphenicol (51\%). ${ }^{21}$ Klebsiella spp. also found to be sensitive to Chloramphenicol where as in other studies it was documented as highly sensitive to Amikacin. ${ }^{16}$ Citrobacter spp. were also found to be resistant to Ampicillin and Cephalexin in 100 percent and 88.9 percent, respectively; whereas it was found to be sensitive to Amikacin and Ceftriaxone in 61.1 percent, each; and Ciprofloxacin, Gentamycin and Chloramphenicol 55.6 percent, each. The study results showed Pseudomonas spp. to be sensitive to Amikacin (72.7\%) which is similar to the study of Iran that documented 54 percent sensitivity of Amikacin and 66.6 percent resistance to Gentamycin. ${ }^{16,22}$ Salmonella spp. were found to be resistant to Ampicillin (100\%) and ceftriaxone (66.7\%), and sensitive to Amikacin and Ciprofloxacin, ((66.7\%), each). Similar resistance pattern for Ampicillin (100\%) was also observed in a study conducted in Kenya. ${ }^{20} \mathrm{~A}$ study in Ethiopia also documented to Amikacin and Ciprofloxacin, ((66.7\%), each). Similar resistance pattern for Ampicillin (100\%) was also observed in a study conducted in Kenya. ${ }^{20}$ This similarity of the increase in the pattern of the antimicrobial resistance in the low income Eastern African countries and Eritrea could be attributed to similar climate change, societal belief and culture, access to antimicrobial drugs and achievement to modern medicine. ${ }^{6,17}$ Sengupta et al., ${ }^{23}$ in their review article documented that even human intervention is a contributing factor for the spread of antimicrobial resistance. ${ }^{23}$ The most commonly used medication during the absence of Ampicillin, Gentamycin, Tetracycline and Amoxicilin is Ciprofloxacin. Currently Ciprofloxacin is started to be sold at drug shops without prescription therefore the development of resistance against this drug is evident. A study in Ethiopia also documented a sensitivity to Ciprofloxacin (100\%), and Amikacin (89.5\%).However, the study documented the highest resistance for Ampicillin (100\%) which is similar to the above studies. ${ }^{24}$ Staphylococcus aureus also found to be sensitive to Rifampicin in 100 percent and to Co - trimoxazole and Chloramphenicol in $(66.7 \%)$ of isolates each; whereas resistance was observed for Nitrofurantoin 100 percent, and Gentamycin, Vancomycin, Oxacillin and Penicillin ((66.7\%), each). A study conducted by Dessie et al., ${ }^{19}$ also documented a sensitivity rate for Gentamycin and Ciprofloxacin ((84.2\%), each), and Tetracycline and Erythromycin in $\left((78.9 \%)\right.$, each) ${ }^{19}$ But a higher resistance rate for penicillin (66.7\%) was observed which is similar to the study conducted in Ethiopia and Sudan that documented 94.7 percent and 91.3 percent, respectively. ${ }^{19,25}$ In this study high antimicrobial resistance could be attributed to inappropriate specimen selection, collection and preparation, improper laboratory and clinical settings preparation, failure to follow infection prevention control guidelines, malpractices that are related to antibiotic misuse which promote antimicrobial drug resistance. ${ }^{26}$ Levy, ${ }^{2}$ in his paper that entitled
"The Challenge of Antibiotic Resistance", concluded that bacteria insensitivity to an antibiotic appears in a person or in a community mainly due to two forces that include prevalence of resistance genes and the extent of antibiotic use. ${ }^{2}$

Therefore, this study has similarity and differences to different studies on antimicrobial resistance that have been done in many. all the above studies revealed a different resistance and susceptibility pattern of antimicrobial drugs that can be due to increased mobility and globalization that are reducing the time needed for antibioticresistant microorganisms to spread simply throughout the world. ${ }^{27-29}$ The core action that helps for fighting the spread of antimicrobial resistance is an improved and appropriate use of antimicrobials.

\section{Conclusion and recommendation}

In general the study results revealed that the isolates were sensitive to Chloramphenicol Amikacin, Ciprofloxacin, Ceftriaxone and Gentamycin; whereas they were resistant to Cephalexin, Ampicillin, Ceftazidime, Nitrofurantoin, Co - trimoxazole, Nalidixic Acid and Tetracycline. This resistance is mainly due to antibiotic misuse that includes people's use of drugs for viral infections buying from pharmacies and drug shops without prescription as self-administered drugs for the treatment of health problems that does not require, like colds and flu; and as growth promoters in animals and fish. ${ }^{30}$

Therefore, this study will help for policy makers and specifically to the drug administration regulatory body to develop policies regarding the rational drug use and proper classification of drugs based on the level of health facility and human resources deployment which is mainly related to professional hierarchy. The study recommends that the practice of rational drug use mainly for the prescription drugs that include antibiotics to be permissible and continuous surveillance for antimicrobial drug sensitivity test to be done at different localities, in order to administer drug that is appropriate for treating disease and reducing the emergence of new resistant bacteria and minimizing the spread of resistant microbial isolates.

\section{Competing interests}

All authors of the study declare that they have no conflict of interests associated with the publication of this paper.

\section{Authors' contribution}

Yemane Seyoum was involved in the designing and proposal writing, interpretation of the study findings, writing and reviewing of the report and final paper preparation. Elias Teages Adgoy was involved, in the designing and proposal writing, in data collection, data analysis and interpretation of the study findings, writing and reviewing of the report and final paper preparation. Kidane Siele was involved in data collection, in the designing and proposal writing, interpretation of the study findings, writing and reviewing of the report and final paper preparation. Mohammed Elfatih was involved in designing and proposal writing of the study, report reviewing and final paper preparation.

Nesterab Gebreleul was involved in data collection.

\section{Acknowledgements}

All authors are thankful to the Ministry of Health, and to the staff of National Health Laboratory, Asmara, Eritrea, for giving permission for the study to be conducted and their cooperation during the data collection period. 


\section{Conflict of interest}

The author declares no conflict of interest.

\section{Funding}

The study was funded by the researchers.

\section{References}

1. Kohanski MA, Dwyer DJ, Collins JJ. How antibiotics kill bacteria: from targets to networks. Nat Rev Microbiol. 2010;8(6):423-435.

2. Levy SB. The challenge of antibiotic resistance. Sci Am. 1998;278(3):4653.

3. Katsikogianni M, Missirlis YF. Concise review of mechanisms of bacterial adhesion to biomaterials and of techniques used in estimating bacteria-material interactions. Eur Cell Mater. 2004;8(3):37-57.

4. Sivagnanam G, Thirumalaikolundusubramanian P, Mohanasundaram J, et al. A survey on current attitude of practicing physicians upon usage of antimicrobial agents in southern part of India. MedGenMed. 2004;6(2):1.

5. Garvey C, McBride T, Nevin L, et al. Antimicrobial Resistance: Is the World UN prepared? PLoS Med. 2016;13(9):e1002130.

6. Omulo S, Thumbi SM, Njenga MK, et al. A review of 40 years of enteric antimicrobial resistance research in Eastern Africa: what can be done better? Antimicrob Resist Infect Control. 2015;4(1):1.

7. Chamoun K, Farah M, Araj G, et al. Surveillance of antimicrobial resistance in Lebanese hospitals: retrospective nationwide compiled data. Int J Infect Dis. 2016;46:64-70.

8. WHO. Antimicrobial resistance: global report on surveillance. Switzerland: World Health Organization; 2014.

9. Ventola CL. The antibiotic resistance crisis: part 1:causes and threats. PT. 2015;40(4):277.

10. Sosa AJ, Byarugaba DK, Amabile-Cuevas CF, et al. Antimicrobial Resistance in Developing Countries. 2010.

11. Coast J, Smith RD, Millar MR. Superbugs: should antimicrobia resistance be included as a cost in economic evaluation? Health Econ 1996;5(3):217-226.

12. Smith RD. Antimicrobial resistance: the importance of developing longterm policy. Bull World Health Organ. 1999;77(10):862.

13. Ministry of Health. Eritrean National Formulary. 2015.

14. Ministry of Health. Eritrean National List of Medicine. 2015.

15. CLSI. Performance Standards for Antimicrobial Susceptibility Testing. 26th ed. USA: CLSI supplement M100S, Clinical and Laboratory Standards Institute; 2016.
16. Jamshidi M, Javadpour S, Eftekhari TE, et al. Antimicrobial resistance pattern among intensive care unit patients. African Journal of Microbiology Research. 2009;3(10):590-594.

17. Laxminarayan R, Duse A, Wattal C, et al. Antibiotic resistance-the need for global solutions. Lancet Infect Dis. 2013;13(12):1057-1098.

18. Alabi AS, Frielinghaus L, Kaba H, et al. Retrospective analysis of antimicrobial resistance and bacterial spectrum of infection in Gabon, Central Africa. BMC Infect Dis. 2013;13(1):455.

19. Dessie W, Mulugeta G, Fentaw S, et al. Pattern of Bacterial Pathogens and Their Susceptibility Isolated from Surgical Site Infections at Selected Referral Hospitals, Addis Ababa, Ethiopia. International Journal of Microbiology. 2016;2016:8.

20. Odhiambo F, Galgalo T, Wences A, et al. Antimicrobial resistance: capacity and practices among clinical laboratories in Kenya, 2013. Pan Afr Med J. 2014;19:332.

21. Abejew AA, Denboba AA, Mekonnen AG. Prevalence and antibiotic resistance pattern of urinary tract bacterial infections in Dessie area, North-East Ethiopia. BMCRes Notes. 2014;7(1):687.

22. Nkang O, Okonko IO, Mejeha OK, et al. Assessment of antibiotics susceptibility profiles of some selected clinical isolates from laboratories in Nigeria. Journal of Microbiology and Antimicrobials. 2009;1(2):019026 .

23. Sengupta S, Chattopadhyay MK, Grossart HP. The multifaceted roles of antibiotics and antibiotic resistance in nature. Front Microbiol. $2013 ; 4: 47$.

24. Lamboro T, Ketema T, Bacha K. Prevalence and Antimicrobial Resistance in Salmonella and Shigella Species Isolated from Outpatients, Jimma University Specialized Hospital, Southwest Ethiopia. Can J Infect Dis Med Microbiol. 2016;2016:4210760.

25. Kangethe SK, Kiiru J, Kabiru EW, et al. Antimicrobial resistance patterns among $E$. coli isolates from children presenting with diarrhoea at a cosmopolitan hospital in Kenya. 2015.

26. Bhandi SH, Khaiser MI. Klebsiella revisited! Endodontology. 2004:1-3.

27. Smith RD, Coast J. Antimicrobial resistance: a global response. Bulletin of the World Health Organization; 2002. 80(2):126-133.

28. Deurenberg RH, Vink C, Kalenic S, et al. The molecular evolution of methicillin-resistant Staphylococcus aureus. Clin Microbiol Infect. 2007;13(3):222-235.

29. McKenna M. Antibiotic resistance:the last resort. Nature. 2013;499(7459):394-396.

30. World Health Organization. Antimicrobial resistance. 2016. 